\title{
Desarrollo estratégico de RECURSOS HUMANOS EN LA UNIVERSIDAD NACIONAL MAYOR DE SAN MARCOS
}

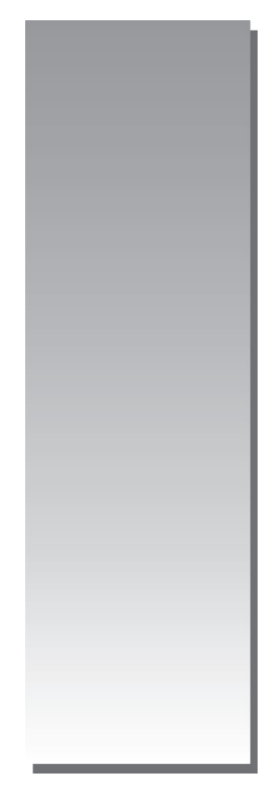

STRATEGic human RESOURCES DEVELOPMENT AT THE NATIONAL UNIVERSity OF SAN MARCOS

Kennedy Narciso Gómez

enrique.angles@tu-berlin.de

[RECEPCIÓN: ABRIL 2016 / CONFORMIDAD: MAYO 2016]

\begin{abstract}
RESUMEN
El campo del desarrollo estratégico de recursos humanos se centra en dos pasos. Primero se considera el planeamiento estratégico; y segundo, el desarrollo estratégico de recursos humanos. En el primer paso, consideramos el pensamiento estratégico adaptado de Michael Porter como herramienta de gestión empresarial que posibilitará la incorporación de los procesos involucrados a la vida cotidiana de la universidad con la participación directa de las facultades y oficinas centrales de línea en el proceso de planeamiento estratégico, con todos los beneficios que puede aportar la aplicación de su conocimiento y experiencia de primera mano al desempeño total de la universidad (Porter, 1982, $\left.1987^{\circ-0}, 1990^{\circ 000}, 1992\right)^{-000}$.

El objetivo central de la investigación es formular un plan de desarrollo estratégico de recursos humanos en la Universidad Nacional Mayor de San Marcos; en este primer paso desarrollamos el diagnóstico de las fuerzas externas (factores económicos, políticos, tecnológicos, culturales, sociales y demográficos), los niveles del entorno empresarial (industria nacional e internacional), el análisis de la industria (modelo de las cinco fuerzas competitivas), el análisis interno (cadena de valor) y el proceso estratégico (formulación, implementación y control). Hemos considerado tres etapas para el marco de formulación de la estrategia: Primera etapa: entrada de datos (matriz de evaluación de factores interno, matriz de evaluación de factores externos, matriz de perfil competitivo). Segunda etapa: etapa comparativa (matriz DOFA: debilidades, oportunidades, fortalezas, amenazas). Tercera etapa: etapa de las decisiones (matriz cuantitativa de planeación estratégica). En el segundo paso se procedió a elaborar el modelo del desarrollo estratégico de los recursos humanos, considerando la visión, misión, fines, políticas, objetivos, identificación de las categorías de decisión estratégicas en el área de recursos humanos y programa de acciones estratégicas. Finalmente, consideramos invertir en el desarrollo estratégico de los recursos humanos de la UNMSM, que posibilitará lograr el éxito de su estrategia para alcanzar su misión.
\end{abstract}

\footnotetext{
Docente investigador del Instituto de Investigación de la Facultad de Ciencias Administrativas, adscrito a la Escuela de Administración, de la UNMSM.

*** Porter, M.E. 1982. Estrategia competitiva. Técnicas para el análisis de los sectores industriales y de la competencia. CECSA. México

䊎米 Porter, M.E. 1987. Ventaja competitiva. Creación y sostenimiento de un desempeño superior. CECSA. México

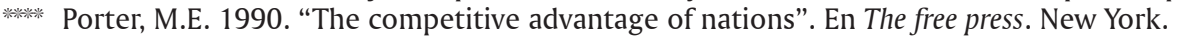

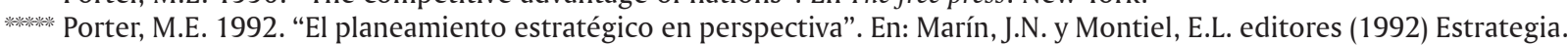
Diseño y ejecución. Asociación Libro Libre. Pp.19-35. San José.
} 
Palabras clave: Desarrollo estratégico de recursos humanos; análisis interno; análisis externo; proceso para la formulación de la estrategia; niveles del entorno estratégico.

\begin{abstract}
The field of strategic human resource development focuses on two steps. First it is considered strategic planning; and second the strategic development of human resources. In the first step, we consider strategic thinking adapted from Michael Porter as business management tool will enable the incorporation of the processes involved in the daily life of the University with the direct participation of the Faculties and Headquarters Line in the strategic planning process with all the benefits it can bring the application of their knowledge and experience firsthand the overall performance of the University. (Porter, 1982, 1987, 1990, 1992).

The main objective of the research is to formulate a plan of strategic development of human resources of the National University of San Marcos; in this first step we develop the diagnosis of external forces (economic, political, technological, cultural, social and demographic), levels of the business environment (industry, national, international), industry analysis (model 5 Competitive Forces), internal analysis (value chain); the strategic process (formulation, implementation, control). We have considered 3 stages to the Framework Strategy Formulation: First step: Data Entry (Evaluation Matrix Matrix internal factors external factors evaluation matrix competitive profile..). Second stage: Stage comparative (SWOT Matrix Weaknesses, opportunities, strengths, threats). Third stage: Stage of decisions (quantitative strategic planning matrix). In the second step we proceeded to develop the Model of Strategic Development of Human Resources, considering the vision, mission, goals, policies, objectives, identifying the categories of strategic decisions in the area of Human Resources, Strategic Action Program. Finally, we consider investing in the Strategic Development of Human Resources of San Marcos, which will enable the success of its strategy to achieve its mission.
\end{abstract}

Keywords: Strategic human development; internal analisis; external analisis; process for the formulation of the estrategy; levels of the strategic evironment.

\section{INTRODUCCIÓN}

La Universidad Nacional Mayor de San Marcos fue fundada el 12 de mayo de 1551 y creada por Real Cédula. Así, de un nivel de primeras letras se dio un salto a la educación superior. La educación intermedia surgió después. En 1571 fue confirmada por Breve del papa Pío V. San Marcos aparece como una Universidad estatal y pontificia, bajo el amparo de ambos poderes. Actualmente está liderada por la Dra. Antonia Florencia Castro Rodríguez, rectora interina de la Universidad Nacional Mayor de San Marcos, a partir del 4 de enero del 2016, en cumplimiento con la Resolución Rectoral № 00001-R-16, de fecha 11 de enero del 2016, concordante con el artículo № 18 de la Constitución Política del Perú y de la Ley Universitaria № 30220, de fecha 8 de julio del 2014. Desde un inicio, la conducción de la universidad se convierte en un asunto político y los cargos directivos fueron ocupados por docentes con ideas conservadoras, que impedían la formación de ideas y conocimientos innovadores. Sin embargo, en cumplimiento de la Ley Universitaria № 30220 , por primera vez mediante voto universal el 3 de abril de 2016 se eligieron 36 miembros de la Asamblea Estatutaria, quienes deberán aprobar un nuevo estatuto para la UNMSM, que permita, con el esfuerzo individual y capacidad de equipo integrada por doce profesores principales, ocho asociados y cuatro auxiliares, y doce estudiantes, en que se incluye un representante de posgrado, iniciar un proceso de innovación radical para lograr el liderazgo institucional; asimismo, nuestra universidad cuenta con un Plan Estratégico Institucional 2012-2021, establecido de manera coherente con el entorno externo e interno de las facultades, en donde se indica la visión de integrar a la universidad a nivel del mundo en el marco de la globalización e internacionalización con las mejores universidades; asimismo, se plantean tres ejes estratégicos fundamentados en la educación superior: educación de calidad e internacionalización; investigación para el desarrollo humano sostenible; y formación humanística y creación cultural. Por otro lado, la proyección de la visión institucional incluye tres pilares: valoración de la continuidad histórica; transformación del mundo; y la identidad nacional y aspiración institucional. 
El problema central de la investigación significa responder ¿en qué medida el desarrollo estratégico influye en la administración de los recursos humanos de la Universidad Nacional Mayor de San Marcos?; esto implica responder a los problemas específicos: a. ¿Cuáles son y en qué medida las amenazas y oportunidades institucionales influyen sobre la administración de recursos humanos de la UNMSM?; b. ¿Cuáles son y en qué medida las fortalezas y debilidades institucionales influyen sobre la administración de recursos humanos de la UNMSM?; c. ¿Cómo y en qué medida la misión, los objetivos y las estrategias actuales ayudaron en la administración de los recursos humanos de la UNMSM?; d. ¿En qué forma la formulación de nuevas misión, objetivos y estrategias ayudarán a mejorar la administración de recursos humanos de la UNMSM?; e. ¿En qué forma las metas, las políticas y las acciones funcionales ayudarán a mejorar la administración de recursos humanos de la UNMSM?; f. ¿Cuál será el costo de implementar un plan de desarrollo estratégico de los recursos humanos de la UNMSM?

Podemos definir el "desarrollo estratégico de recursos humanos" como el esfuerzo continuo reflejado en el plan de recursos humanos, que comprende estrategias que garantizan la adecuada y oportuna cobertura de recursos humanos capaces, diseñando para ello procesos especializados de selección, promoción y colocación, desarrollo del personal, evaluación del desempeño, recompensas, relación dirección/empleados, etc.; con el fin de alcanzar un desarrollo armónico y sostenido. Todo ello inserto en el plan estratégico general de la institución.

\section{MÉTODO}

Empleamos los métodos "inductivo-deductivo", referida a las variables "desarrollo estratégico" y "recursos humanos". Empleamos una muestra de 180 trabajadores, de los cuales 125 son docentes y 55 no docentes. Este tamaño de muestra se obtuvo para un nivel de confianza de $95 \%$, un error muestral de $5 \% \mathrm{y}$, dado que no existen antecedentes sobre el tema de la investigación, una proporción real de éxito de $50 \%$.

\footnotetext{
Narciso, K. 2004. "Desarrollo estratégico de recursos humanos en la Universidad del Callao". Escuela de Posgrado de la Universidad Inca Garcilaso de la Vega. Tesis para optar el grado de Doctor en Administración. Lima.
}

(Narciso, 1992)" acerca de la importancia de la gestión estratégica del personal para colocar cuadros ejecutivos en los puestos claves de la gestión universitaria, a fin de mejorar la productividad del personal en la Universidad Nacional Daniel Alcides Carrión de Cerro de Pasco. Complementada y mejorada con mi tesis doctoral. En lo que sigue, a partir de este punto hasta el punto 4.3 de la etapa decisoria, presentamos un extenso resumen de las ideas de David y adaptado por el investigador para la UNMSM (1996)

Tabla № 1 . Objetivos de largo y corto plazos por categorías de toma de decisiones estratégicas de la UNMSM.

\begin{tabular}{lc}
\hline & \multicolumn{2}{c}{ OBJETIVOS } \\
\cline { 2 - 2 } CATEGORÍA & \multicolumn{2}{c}{ CORTO PLAZO $\quad$ LARGO PLAZO }
\end{tabular}

\begin{tabular}{|c|c|c|}
\hline $\begin{array}{l}\text { Selección, } \\
\text { promoción y } \\
\text { colocación }\end{array}$ & $\begin{array}{l}\text { Poner en práctica un } \\
\text { plan de desarrollo in- } \\
\text { dividual para todo el } \\
\text { equipo. }\end{array}$ & $\begin{array}{l}\text { Desarrollarelpersonal } \\
\text { actual a través de un } \\
\text { entrenamiento enel } \\
\text { puestoy del reentre- } \\
\text { namientoparaocupar } \\
\text { posiciones futuras. }\end{array}$ \\
\hline $\begin{array}{l}\text { Evaluacióndel } \\
\text { desempeño }\end{array}$ & $\begin{array}{l}\text { Daralosdirectivosun } \\
\text { sistemadeevaluación } \\
\text { con el que se sientan } \\
\text { cómodos. }\end{array}$ & $\begin{array}{l}\text { Cambiarlaevaluación } \\
\text { hacia un sistema de } \\
\text { asesoramiento. }\end{array}$ \\
\hline Recompensas & $\begin{array}{l}\text { Reforzarladiferencia- } \\
\text { ciónentrelosejecuto- } \\
\text { res. }\end{array}$ & $\begin{array}{l}\text { Continuardiferencián- } \\
\text { dolas sin alienar al } \\
\text { ejecutor promedio. }\end{array}$ \\
\hline $\begin{array}{l}\text { Desarrollo de } \\
\text { personal }\end{array}$ & $\begin{array}{l}\text { Preparar directivos y } \\
\text { personal para el cam- } \\
\text { bianteambienteuniver- } \\
\text { sitario. }\end{array}$ & $\begin{array}{l}\text { Incrementarlapartici- } \\
\text { pacióndelmercado,la } \\
\text { calidad del servicioy } \\
\text { la productividad. }\end{array}$ \\
\hline $\begin{array}{l}\text { Relaciones } \\
\text { dirección/em- } \\
\text { pleados }\end{array}$ & $\begin{array}{l}\text { Motivacióndelpersonal } \\
\text { en todos los niveles. }\end{array}$ & $\begin{array}{l}\text { Desarrollarrelaciones } \\
\text { de armonía, sobre la } \\
\text { basedeunincremen- } \\
\text { todelos beneficiosy } \\
\text { delaparticipacióndel } \\
\text { mercado. }\end{array}$ \\
\hline
\end{tabular}

Fuente: Grupo de Investigación.

\footnotetext{
橉 Narciso, K. 1998. "Gestión de recursos humanos en la UNDAC". Escuela de Posgrado de la Universidad Nacional Mayor de San Marcos. Tesis para optar al grado de Magíster en Administración, p. 19. Lima.

***** DAVID, F. R. 1996. Conceptos de administración estratégica. 6a. ed. Editorial Prentice-Hall Internacional. México.
} 
Marco general para la formulación de la estrategia

Primera etapa: ENTRADA DE DATOS

Matriz de evaluación de factor interno UNMSM-Lima, 2015

\begin{tabular}{|c|c|c|c|}
\hline Tabla № 2. Factores críticos de éxito & Peso & Calificación & Total ponderado \\
\hline \multicolumn{4}{|l|}{ FORTALEZAS } \\
\hline $\begin{array}{l}\text { Existenciadeprocesosdeplanestratégicoinstitucional2011-2021 } \\
\text { en ejecución }\end{array}$ & 0.15 & 4 & 0.6 \\
\hline Nueva currícula de estudio & 0.10 & 3 & 0.3 \\
\hline Docentes en proceso de actualizacióny capacitación continua & 0.15 & 4 & 0.6 \\
\hline Equipamiento continuo de laboratorios & 0.10 & 3 & 0.3 \\
\hline Acceso a internet, aulas virtuales y biblioteca virtual & 0.15 & 4 & 0.6 \\
\hline \multicolumn{4}{|l|}{ DEBILIDADES } \\
\hline Enseñanza libresca & 0.10 & 1 & 0.1 \\
\hline Bajas remuneraciones del personal docente y administrativo & 0.05 & 1 & 0.05 \\
\hline Existenciadepocosdocentescondoctoradosyposdoctorados & 0.10 & 1 & 0.1 \\
\hline Se realiza muy poca investigación con-con, sin-sin & 0.05 & 1 & $0 . .5$ \\
\hline Bajo nivel académico de los docentes y estudiantes & 0.05 & 1 & 0.05 \\
\hline Total & 1.00 & & 2.75 \\
\hline
\end{tabular}

Fuente: Grupo de investigadores

La matriz de evaluación de factores externos de la UNMSM-Lima, 2015.

\begin{tabular}{lccc}
\hline \multicolumn{1}{c}{ Tabla $\mathrm{n}^{\circ}$ 3. Factor externo clave } & Ponderación & Clasificación & Resultado Ponderado \\
\hline $\begin{array}{l}\text { Existenciadeinstitucionesnacionaleseinternacionalesconlasque } \\
\text { podrían establecerse convenios de cooperación. }\end{array}$ & 0.20 & 4 & 0.8 \\
\hline $\begin{array}{l}\text { Demandadeproyectos deinvestigaciónproductivosenel país. } \\
\text { Demanda creciente porformación universitaria (nivelestatal). }\end{array}$ & 0.10 & 4 & 0.4 \\
\hline Crisis económica y limitada creación de empleos. & 0.15 & 1 & 0.8 \\
\hline $\begin{array}{l}\text { IncapacidaddelEstadoparasatisfacernecesidadesdeuniversida- } \\
\text { des estatales. }\end{array}$ & 0.05 & 1 & 0.15 \\
\hline $\begin{array}{l}\text { DesinterésydesconfianzadelacomunidadempresarialydelEsta- } \\
\text { doenlasuniversidadesestatalesparagenerarproyectosconjuntos. }\end{array}$ & 0.10 & 1 & 0.05 \\
\hline $\begin{array}{l}\text { Deterioro generalizado de la moral pública. } \\
\text { Demandadeprofesionalesmejorformadosymáscompetitivos. }\end{array}$ & 0.15 & 2 & 0.10 \\
\hline \begin{tabular}{l} 
TOTAL \\
\hline
\end{tabular}
\end{tabular}

Fuente: grupo de investigadores 
Observando el resultado de la matriz de evaluación de factores externos, se puede concluir que la UNMSM se encuentra en un ambiente en el que enfrenta más oportunidades que amenazas.

Matriz de perfil competitivo UNMSM - Lima, 2015.

\begin{tabular}{|l|c|c|c|c|c|c|c|c|c|c|c|c|c|}
\hline \multirow{2}{*}{ TablaN4.Factoresclavesdeéxito } & \multirow{2}{*}{ Ponderación } & \multicolumn{2}{|c|}{ Univ. 1} & \multicolumn{2}{|c|}{ Univ. 2 } & \multicolumn{2}{c|}{ Univ. 3 } & \multicolumn{2}{c|}{ Univ. 4 } & \multicolumn{2}{c|}{ Univ. 5} & \multicolumn{2}{c|}{ Univ. 6} \\
\hline & & Cla- & Rp & Clasif & Rp & Clasif & Rp & Clasif & Rp & Clasif & Rp & Clasif & Rp \\
\hline Servicio al cliente & 0.15 & 1 & 0.15 & 1 & 0.15 & 2 & 0.30 & 1 & 0.15 & 1 & 0.15 & 1 & 0.15 \\
\hline Prestigio institucional & 0.15 & 1 & 0.15 & 2 & 0.30 & 3 & 0.45 & 2 & 0.30 & 1 & 0.15 & 3 & 0.45 \\
\hline Precio & 0.25 & 3 & 0.75 & 3 & 0.75 & 3 & 0.75 & 3 & 0.75 & 3 & 0.75 & 3 & 0.75 \\
\hline Calidadacadémicaeinvestigación & 0.15 & 1 & 0.15 & 2 & 0.30 & 4 & 0.60 & 1 & 0.15 & 1 & 0.15 & 2 & 0.30 \\
\hline Superioridad tecnológica & 0.10 & 1 & 0.10 & 3 & 0.30 & 3 & 0.30 & 2 & 0.20 & 1 & 0.10 & 2 & 0.20 \\
\hline Fortaleza financiera & 0.20 & 1 & 0.20 & 2 & 0.40 & 2 & 0.40 & 2 & 0.40 & 1 & 0.20 & 2 & 0.40 \\
\hline Resultados totales & 1.00 & & 1.50 & & 2.20 & & 2.80 & & 1.95 & & 1.50 & & 2.25 \\
\hline
\end{tabular}

Fuente: grupo de investigadores

(Univ. 1: UNAC; Univ. 2: UNI; Univ. 3: U. N. Agraria La Molina; Univ. 4: U. N. Federico Villareal; Univ. 5: U. N. de Educación Enrique Guzmán y Valle - La Cantuta; Univ. 6: UNMSM)

\section{Segunda etapa: LA ETAPA COMPARATIVA}

Matriz DOFA - Lima, 2015.

\begin{tabular}{|c|c|c|}
\hline & \begin{tabular}{|l|} 
FORTALEZAS (F) \\
Precio. \\
Existenciadeplaneamientoestratégicoyop- \\
erativo en ejecución. \\
Nuevos currículos de estudio. \\
Docentesenprocesodeactualizaciónyca- \\
pacitación continuo. \\
Equipamientocontinúo delaboratorios. \\
Generacióndegestióndelconocimiento. \\
Acceso a Internet. \\
Aulas virtuales modernas. \\
Informes de investigación. \\
Acreditacióndelacalidadinstitucionalyde \\
facultades.
\end{tabular} & $\begin{array}{l}\text { DEBILIDADES (D) } \\
\text { Enseñanza libresca } \\
\text { Existenciadepocosdocentescon } \\
\text { doctorados y posdoctorados. } \\
\text { Bajonivelacadémicodedocentesy } \\
\text { estudiantes. } \\
\text { Ascenso limitado de docentes. } \\
\text { Servicio al cliente } \\
\text { Prestigio institucional } \\
\text { Calidadacadémicaeinvestigación } \\
\text { Tecnología } \\
\text { Fortaleza financiera }\end{array}$ \\
\hline $\begin{array}{l}\text { OPORTUNIDADES }(\mathrm{O}) \\
\text { Existenciadeinstitucionesnacionaleseinternacio- } \\
\text { nalesconlasquepodríanestablecerseconveniosde } \\
\text { cooperación. } \\
\text { Demandadeproyectosdeinvestigaciónproductivos } \\
\text { por empresas en el país. } \\
\text { Demandacrecienteporformacióntécnicayengestión } \\
\text { empresarial. } \\
\text { Demanda alta por la educación superior. }\end{array}$ & \begin{tabular}{|l|} 
ESTRATEGIAS (FO) \\
Establecerprogramas deinvestigaciónen \\
convenioconempresasyentidadespúbli- \\
cas. \\
Establecercentrosdeproduccióndebienes \\
y servicios a cargo de docentes y estudi- \\
antes. \\
Desarrollarprogramas deextensión bajo \\
responsabilidaddedocentesyestudiantes.
\end{tabular} & $\begin{array}{l}\text { ESTRATEGIAS (DO) } \\
\text { Establecerconvenios decooper- } \\
\text { aciónydeasistenciaconinstitucio- } \\
\text { nes nacionales y extranjeras. } \\
\text { Desarrollarprogramasorgánicos } \\
\text { deformación,capacitaciónyentre- } \\
\text { namiento,paradocentesypersonal } \\
\text { administrativo. }\end{array}$ \\
\hline $\begin{array}{l}\text { AMENAZAS (A) } \\
\text { Crisiseconómicay limitada creación deempleos. } \\
\text { IncapacidaddelEstadoparasatisfacernecesidades } \\
\text { de universidades estatales. } \\
\text { Desinterésydesconfianzadelacomunidadempre- } \\
\text { sarialydelEstadoenlasuniversidadesestatalespara } \\
\text { generar proyectos conjuntos. } \\
\text { Deterioro generalizado de la moral pública. } \\
\text { Demandadeprofesionalesmejorformadosymás } \\
\text { competitivos. } \\
\text { Ley Universitaria }\end{array}$ & $\begin{array}{l}\text { ESTRATEGIAS (FA) } \\
\text { Desarrollarprogramasdeformación/capaci- } \\
\text { tación paramypes, a cargo de docentesy } \\
\text { estudiantes. }\end{array}$ & $\begin{array}{l}\text { ESTRATEGIAS (DA) } \\
\text { Manteneradocentesmayoresde } \\
70 \text { años. } \\
\text { Implementar la Escuela de Pos- } \\
\text { grado con posdoctorados. }\end{array}$ \\
\hline
\end{tabular}

Fuente: grupo de investigadores 
Tercera etapa: La etapa decisoria

Matriz cuantitativa de planificación estratégica. México, 1996.

\begin{tabular}{|c|c|c|c|c|c|}
\hline \multirow{3}{*}{ FACTORES CRÍTICOS DE ÉXITO } & \multirow{4}{*}{ PESO } & \multicolumn{4}{|c|}{ ESTRATEGIAS ALTERNATIVAS } \\
\hline & & \multicolumn{2}{|c|}{$\begin{array}{l}\text { JOINT VENTURE EN } \\
\text { EUROPA }\end{array}$} & \multicolumn{2}{|c|}{$\begin{array}{l}\text { JOINT VENTURE EN } \\
\text { ASIA }\end{array}$} \\
\hline & & P.A. & T.P.A. & P.A. & T.P.A. \\
\hline \multicolumn{5}{|l|}{ OPORTUNIDADES } & \\
\hline 1. Unificación de Europa Occidental. & 0.10 & 4 & 0.40 & 2 & 0.20 \\
\hline 2. Mayor conciencia de salud para elegir alimentos. & 0.15 & 3 & 0.45 & 3 & 0.45 \\
\hline 3. Economías de libre comercio nacientes en Asia. & 0.10 & 2 & 0.20 & 4 & 0.40 \\
\hline 4. Demanda de sopas aumenta $10 \%$ al año. & 0.15 & 3 & 0.45 & 3 & 0.60 \\
\hline 5. TLC México - EUA. & 0.05 & - & - & - & - \\
\hline \multicolumn{6}{|l|}{ AMENAZAS } \\
\hline 1. Ingreso de alimentos aumenta $1 \%$ al año. & 0.10 & 3 & 0.30 & 3 & 0.30 \\
\hline 2. Alimentos Banquet de ConAgra tienen $27.4 \%$ del mercado. & 0.05 & - & - & - & - \\
\hline 3. Economías inestables en Asia. & 0.10 & 4 & 0.40 & 1 & 0.10 \\
\hline 4. Envases de latón no biodegradables. & 0.05 & - & - & - & - \\
\hline 5. Valor bajo del dólar. & 0.15 & 4 & 0.60 & 2 & 0.30 \\
\hline \multicolumn{6}{|l|}{ FORTALEZAS } \\
\hline 1. Incremento de utilidades $30 \%$. & 0.10 & 3 & 0.30 & 3 & 0.30 \\
\hline 2. Nueva división para América del Norte. & 0.10 & - & - & - & - \\
\hline 3. Nuevas sopas saludables tienen éxito. & 0.10 & 4 & 0.40 & 2 & 0.20 \\
\hline 4.ParticipacióndemercadodealimentosSwansonsubióa25.1\%. & 0.05 & 3 & 0.15 & 3 & 0.15 \\
\hline $\begin{array}{l}\text { 5. Unquintodelosbonosdegerentesbasadossobrelosresultados } \\
\text { generales de la corporación. }\end{array}$ & 0.05 & - & - & - & - \\
\hline 6. Aprovechamiento de capacidad pasó de 60 a $80 \%$. & 0.15 & 3 & 0.45 & 3 & 0.45 \\
\hline \multicolumn{6}{|l|}{ DEBILIDADES } \\
\hline 1. Ventas de Pepperidge Farm cayeron $7 \%$. & 0.05 & - & - & - & - \\
\hline 2. Costo de reestructuración $\$ 302$ millones. & 0.05 & - & - & - & - \\
\hline 3. La empresa está perdiendo dinero en Europa. & 0.15 & 2 & 0.30 & 3 & 0.45 \\
\hline 4. Demora en globalización de la empresa. & 0.15 & 4 & 0.60 & 3 & 0.45 \\
\hline $\begin{array}{l}\text { 5. Margendeutilidadde8.4\%eslamitaddel promediodelaindus- } \\
\text { tria. }\end{array}$ & 0.10 & 3 & 0.30 & 3 & 0.30 \\
\hline TOTAL & & & 5.30 & & 4.65 \\
\hline
\end{tabular}

Fuente: DAVID, F.R. 1996. Conceptos de administración estratégica. 6a. ed. Editorial Prentice-Hall Internacional. México. 


\section{Diagnóstico externo: factores y niveles}

Gráfico 1. Diagnóstico externo: interacción con SUNEDU.

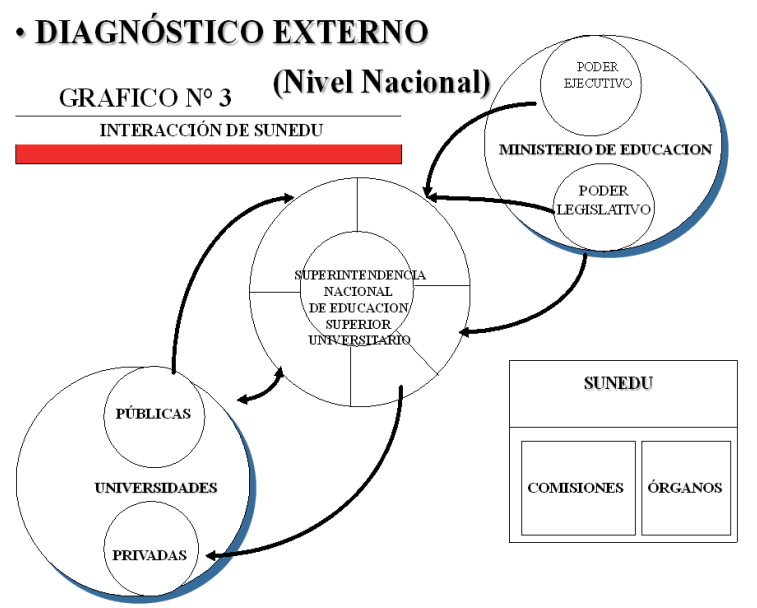

Fuente: Grupo de investigadores

Gráfico 2. Diagnóstico externo: análisis industrial.

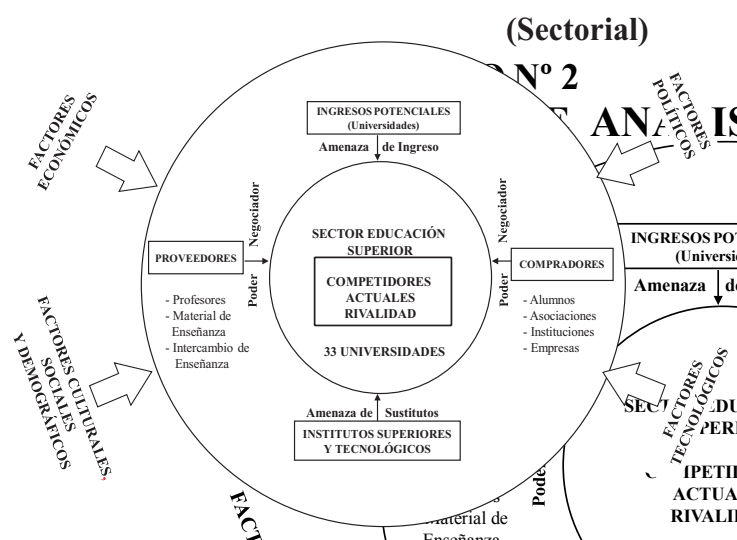

Fuente: grupo de investigadores

\section{RESULTADOS}

PLAN DE DESARROLLO DE RECURSOS HUMANOS 2016-2030 DE LA UNIVERSIDAD NACIONAL MAYOR DE SAN MARCOS

1. Selección de estrategias de la UNMSM

\subsection{Estrategia genérica}

La universidad deberá adecuarse a los cambios ambientales tendientes a alcanzar a sus clientes una formación única y un servicio único, por lo que la alternativa estratégica genérica que recomendamos es el liderazgo en costos.

1.2. Estrategia específica
Se concluye el orden en que deberían implantarse las estrategias seleccionadas en la matriz DOFA (ver cuadro № 5), presentamos el primero: Estrategia №5 "Desarrollar programas orgánicos de formación, capacitación y entrenamiento, para docentes y personal administrativo".

2. Visión y misión del área de recursos humanos

2.1. Visión

Ser competitivos en el largo plazo, con un equipo eficaz de recursos humanos que garantice el desarrollo de la universidad.

\subsection{Misión}

Contribuir al logro y mantenimiento de un clima laboral propicio para el logro de los objetivos institucionales mediante una adecuada administración de los sistemas de personal, así como para la formación de mejores cuadros de recursos humanos satisfechos, identificados con la universidad, con una integración plena, con un bienestar acorde a la realidad y una adecuada capacitación con el fin de que mejoren continuamente en concordancia con el avance científico y tecnológico.

\subsection{Objetivos generales}

a. Asegurar la cobertura adecuada y oportuna de los cuadros de recursos humanos de la universidad, a través de un proceso de desarrollo de las fuentes de personal interno y externo.

b. Cubrir los puestos claves con personal que haya demostrado un efectivo potencial de desarrollo.

c. Establecer sistemas de capacitación y evaluación continuo para medir el grado de progreso de conocimiento del personal y su adaptación a los objetivos, estrategias y políticas de la universidad (ver cuadro $\mathrm{N}^{\circ} 1$ ).

\subsection{Programa de acciones estratégicas}

2.4.1. Selección, promoción y colocación

2.4.2. Desarrollo del personal

2.4.3. Evaluación del desempeño

\subsubsection{Recompensas}

2.4.5. Relación dirección/empleados

2.4.6. Infraestructura de apoyo, académica y operativa

2.4.7. Control 


\subsubsection{Programa y costos estimados}

\section{a. DISCUSIÓN}

El conocimiento de las capacidades y habilidades de su personal, hará que la institución planifique mejor el uso y el desarrollo de sus recursos humanos: si se definen las tareas correctas, se ponen a las personas apropiadas a cargo y se vuelve a ellas con el sistema de recompensa adecuado, no se necesita ser un buen gerente para obtener resultados excelentes. Por tanto, en función de los recursos con que cuenta la UNMSM, es importante implantar las estrategias identificadas en la matriz DOFA, en el orden de prioridad presentada como estrategias específicas en el presente trabajo, mostraremos el primero:

1. E5: Desarrollar programas orgánicos de formación, capacitación y entrenamiento, para docentes y personal administrativo.

2. Desarrollar actividades apropiadas para poner en práctica dichas estrategias, en función de las políticas de la UNMSM.

Podemos mencionar que el éxito de Sony da validez al punto de vista de Akio Morita, quien dice que "el recurso más importante de una organización es la gente que proporciona su trabajo, su talento, su creatividad e impulso a la organización [...] El mundo del trabajo nos hace un examen cada día. El desempeño tiene que ser continuo, y un error no se refleja en un examen reprobado sino en los costos de la compañía [...] Todo el proceso de reclutamiento, selección, capacitación y evaluación de los empleados, se construye sobre la premisa de que los empleados son la parte más valiosa de la compañía”.

\section{b. CONCLUSIONES}

1. De acuerdo con las matrices de perfil competitivo y de evaluación de factores internos, la UNMSM tiene más fortalezas que debilidades y se encuentra en una posición competitiva frente a otros centros de educación superior pertenecientes al sector.

2. Se han identificado las siguientes estrategiasfortalezas- oportunidades:

- Establecer programas de investigación en convenio con empresas y entidades públicas.

- Establecer centros de producción de bienes y servicios a cargo de docentes y estudiantes.
- Desarrollar programas de extensión bajo responsabilidad de docentes y estudiantes.

3. Se identificaron las siguientes estrategiasfortalezas-amenazas:

- Desarrollar programas de formación/capacitación para mypes, a cargo de docentes y estudiantes.

4. Se identificaron las siguientes estrategiasdebilidades-oportunidades:

- Establecer convenios de cooperación y de asistencia con instituciones nacionales y extranjeras.

- Desarrollar programas orgánicos de formación, capacitación y entrenamiento, para docentes y personal administrativo.

5. Se identificaron las siguientes estrategiasdebilidades-amenazas:

- Mantener a docentes mayores de 70 años.

- Implementar la Escuela de posgrado con posdoctorados.

\section{REFERENCIAS BIBLIOGRÁFICAS}

David, F. R. 1996. Conceptos de administración estratégica. $6^{a}$. ed. Editorial Prentice-Hall Internacional. México.

Narciso, K. 1998. "Gestión de recursos humanos en la UNDAC”. Lima, Escuela de Posgrado de la Universidad Nacional Mayor de San Marcos. Tesis para optar al grado de Magíster en Administración.

Narciso, K. 2004. "Desarrollo estratégico de recursos humanos en la Universidad del Callao". Lima, Escuela de Posgrado de la Universidad Inca Garcilaso de la Vega. Tesis para optar el grado de Doctor en Administración.

Porter, M.E. 1982. Estrategia competitiva. Técnicas para el análisis de los sectores industriales y de la competencia. México, CECSA.

Porter, M.E. 1987. Ventaja competitiva. Creación y sostenimiento de un desempeño superior. CECSA. México.

Porter, M.E. 1990. "The competitive advantage of nations". En The Free Press. New York.

Porter, M. 1992. "El planeamiento estratégico en perspectiva”. En: Marín, J. N. y Montiel, E. L. editores 1992. Estrategia. Diseño y ejecución. Asociación Libro Libre. San José. Pp.19-35. 